\title{
Radiation damage study of organic molecules via laser-free ultrafast transmission electron microscopy
}

Hyeokmin Choe ${ }^{1}$, Eric Montgomery ${ }^{2}$, Ilya Ponomarev ${ }^{3}$, June Lau ${ }^{4}$, Yimei Zhu ${ }^{5}$, Yubin Zhao ${ }^{3}$, Ao Liu ${ }^{3}$, Alexei Kanareykin ${ }^{3}$ and Chunguang Jing ${ }^{3}$

${ }^{1}$ National Institute of Standards and Technology, United States, ${ }^{2}$ Euclid Techlabs, United States, ${ }^{3}$ Euclid Techlabs LLC, United States, ${ }^{4}$ National Institute of Standards and Technology, Gaithersburg, Maryland, United States, ${ }^{5}$ Department of Condensed Matter Physics and Materials Science, Brookhaven National Laboratory, Upton, NY, USA, United States

* Corresponding author: hyeokmin.choe@nist.gov

Considerable debate has arisen in the transmission electron microscopy (TEM) community over recent evidence of a higher radiation damage threshold by ultrafast gating of the electron beam. In TEM, the interaction of electrons with beam sensitive materials such as organic and biological macromolecules result in electron radiation damage, which continues to be a major limiting factor for acquiring structure of macromolecules even though many reduction methods have been developed [1]. Mitigation historically used ultra-low beam current and sample cryoprotection; ultrafast pulsed electron beams recently have shown promise but without exploration of pulse tuning.

In this study, we present a revolutionary new approach and compelling data via a novel and practical implementation of the RF-based laser-free ultrafast stroboscopic TEM to study mitigation of radiation damage in organic molecules. We have performed the effects of radiation damage experiments for $\mathrm{C}_{36} \mathrm{H}_{74}$ paraffin as a model of organic molecules using a new ultrafast stroboscopic transmission electron microscope (a modified JEM-3010) in NIST [2-5]. We practically measure the beam current and the illuminated area, acquire a set of diffraction patterns over time from a specific crystal, and monitor the integrated intensities of the 110 Bragg peaks in order to measure the critical dose $\left(D_{c}\right)$ (see Figure 1). The data set was made possible by rapid data acquisition in the single-electron low-dose regime using the $\mathrm{GHz}$ stroboscopic system, which is nearly three orders of magnitude faster than $\sim 200 \mathrm{kHz}$ laser-based systems. This work is that the first to tune electron dose rates smoothly from continuous beam to pulsed beam (by varying duty cycle from $100 \%$ to $10 \%$ ), the first using the same electron emitter and column optics for continuous and pulsed experiments with $\mathrm{LaB}_{6}$ thermionic cathodes, helps to gain additional physical insight into the timescales associated with relaxation processes. The critical dose of the organic specimen for gated beam at $5.2 \mathrm{GHz}$ and $10 \mathrm{pA}$ beam current improves by approximately a factor of 1.5 versus continuous beam at room temperature. This improvement agrees with and is similar that reported by laserbased ultrafast TEM [1]. While debate will continue over the physics of the damage process improved by ultrafast gating, we believe this work offers a powerful new method to probe radiation damage physics and a substantial body of evidence in support of ultrafast stroboscopic mitigation of radiation damage in macromolecules. We anticipate future application of this new technology to cryogenic electron microscopy (CryoEM) and microcrystal electron diffraction (MicroED), paving the way for a new class of highly tunable ultrafast stroboscopic system in structural biology, single particle imaging, and low-dose TEM as an electron dose metering device. 

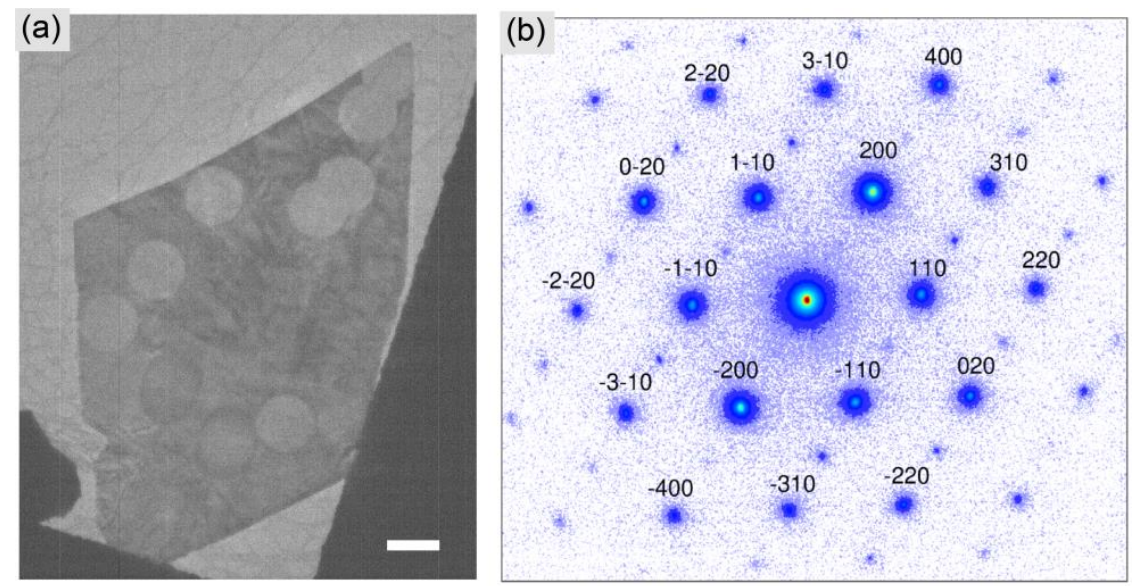

(c)

(d)
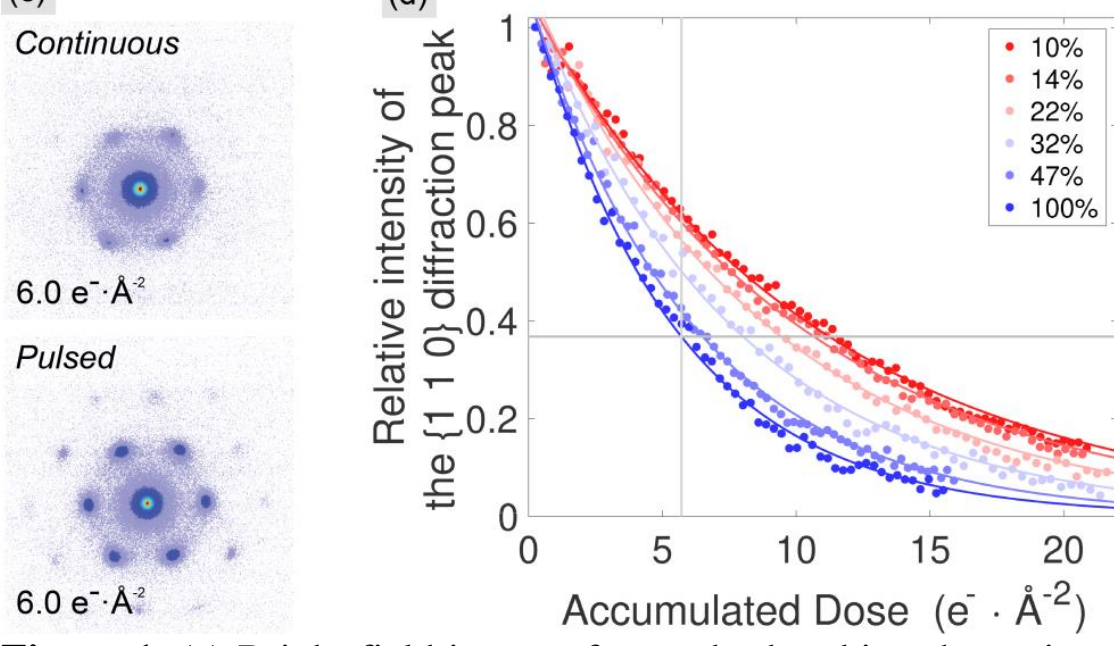

$6.0 \mathrm{e}^{-} \cdot \AA^{-2}$

Accumulated Dose $\left(e^{-} \cdot \AA^{-2}\right)$

Figure 1. (a) Bright-field image of an orthorhombic $n$-hexatriacontane $\mathrm{C}_{36} \mathrm{H}_{74}$ crystal acquired at $600 \mathrm{x}$ magnification. The circles show the electron beam damaged crystal. Scale bar $=5 \mu \mathrm{m}$. (b) Indexed [001] crystallographic zone axis diffraction patterns acquired with a length of $30 \mathrm{~cm}$. (c) Selected continuousand pulsed-beam diffraction patterns of a paraffin crystal with the $\{110\}$ family Bragg peaks used to generate the fading curves in panel (d) highlighted (d) Fading curves of the diffraction patterns and nonlinear exponential decaying fits as a function of different duty cycles of the pulsed beam at $5.2 \mathrm{GHz}$ repetition rate for the same dose rate $\left(0.2546 \mathrm{e}^{-} / \AA^{2} /\right.$ frame $)$. The grey guideline indicates a critical dose $\left(\mathrm{D}_{\mathrm{c}}\right)$.

\section{References}

[1] VandenBussche, et al., Nano Letters. 19, (2019) 6687. DOI:10.1021/acs.nanolett.9b03074

[2] J W Lau, et al., Rev. Sci. Instr. 91 (2020) 021301. DOI:10.1063/1.5131758

[3] C Jing, et al., Ultramicroscopy, 207 (2019) 112829. DOI: 10.1016/j.ultramic.2019.112829

[4] 2020 Microscopy Innovation Award (Microscopy Today)

[5] 2019 R\&D100 Award for “Affordable Laser-free Retrofittable Stroboscopic Solution for Ultra-fast Electron Microscopy” (R\&D 100 Magazine) 